\title{
Quarkonia in STAR
}

\section{Grazyna Odyniec for the STAR Collaboration*}

Lawrence Berkeley National Laboratory, Berkeley, CA 94620, USA

E-mail: G_odyniec@lbl.gov

The results on $\Psi$ and $\Upsilon$ production in $\mathrm{p}+\mathrm{p}, \mathrm{d}+\mathrm{Au}, \mathrm{Cu}+\mathrm{Cu}$ and $\mathrm{Au}+\mathrm{Au}$ collisions obtained by the STAR experiment at the Relativistic Heavy Ion Collider (RHIC) are presented. Studies of the quarkonia production in ultrarelativistic heavy ion collisions are expected to provide valuable information on properties of the dense nuclear medium produced in these collisions as the production is believed to be effected by the Debye color screening of the potential between the heavy quarks. Lattice QCD calculations imply that a sequential suppression of quarkonia states may indicate the temperature of the quark-gluon plasma (QGP) produced in the collisions. The suppression is measured via ratio of production in A+A to properly scaled with the number of collisions yield in $\mathrm{p}+\mathrm{p}\left(R_{A A}\right)$. Those measurements could be complicated by presence of cold nuclear matter effects, which in general used to be studied with $\mathrm{p}+\mathrm{A}$ collisions, but in case of the STAR experiment are investigated in $\mathrm{d}+\mathrm{A}$ interactions. Preliminary results show that $R_{d A}$ in $\mathrm{d}+\mathrm{Au}$ collisions is consistent with binary scaling.

XVIII International Workshop on Deep-Inelastic Scattering and Related Subjects, DIS 2010 April 19-23, 2010

Firenze, Italy

\footnotetext{
* Speaker.
} 


\section{Introduction}

Quarkonium suppression, originally proposed more than 20 years ago [1], in time became a classic signature of quark-gluon plasma formation. It is determined by binding energy of quarkonia states and temperature reached in plasma. With increasing temperature the different quarkonia states "melt" sequentially as a function of their binding energy strength: the most loosely bound state disappears first, while the ground state, last. Dissociation points of the different quarkonia states provide a way to measure the temperature of the medium.

The first results on quarkonium suppression from RHIC show the same level of J/ $\Psi$ suppression as observed in CERN SPS experiments (NA50 and NA60), in spite of the fact that the center-of-mass energy at RHIC is an order of magnitude higher than at CERN SPS. This pointed to the possibility that a new mechanism(s), countering the original suppression, might be involved at RHIC energies. The first candidate considered was the recombination process of charmed quarks in the later stages of the collisions [2]. Also other processes like decay feed-down of higher stated ( $\Psi$ ' and $\chi_{c}$ ), gluon and heavy quark fragmentation processes, energy loss, absorption by co-movers and cold matter effects, need to be studied systematically in order to understand the fraction of direct production and magnitude of suppression.

The Upsilon states are particularly interesting as they provide much less unambiguous measurements than $\mathrm{J} / \Psi$. In the case of Upsilon, the complications related to the contributions from regeneration and feed-down processes are negligible at RHIC energies. However, the cross-section for $\Upsilon$ production is very small that the measurement becomes a challenge.

Note that presently, there is no convincing model of quarkonium production explaining available data even for the simplest case of $p+p$ collisions. Therefore, the detailed studies of quarkonia production and possible suppression in $\mathrm{p}+\mathrm{p}, \mathrm{p}+\mathrm{A}$ and $\mathrm{A}+\mathrm{A}$ is essential.

\section{Experiment}

In this paper the STAR measurements of $\mathrm{J} / \Psi$ and $\Upsilon(1 \mathrm{~S}+2 \mathrm{~S}+3 \mathrm{~S})$ production at mid-rapidity $(-1<\eta<1$, with full azimuthal coverage $)$ in $\mathrm{p}+\mathrm{p}, \mathrm{d}+\mathrm{Au}, \mathrm{Cu}+\mathrm{Cu}$ and $\mathrm{Au}+\mathrm{Au}$ at $\sqrt{s_{N N}}=200 \mathrm{GeV}$ are presented. The decay channel used for reconstruction in all cases is: $\mathrm{J} / \Psi, \Upsilon \rightarrow e^{+}+e^{-}$due to the large electron acceptance of the STAR Time Projection Chamber (TPC) and the electron trigger capabilities of Barrel Electromagnetic Calorimeter (BEMC). In STAR both TPC and BEMC are used for electron identification. At high $\mathrm{p}_{t}$ the BEMC is used for on-line triggering to enrich sample of electrons.

\section{Results}

Figure 1(left) [3] shows the invariant mass distributions for the high- $\mathrm{p}_{t}$ di-electron pairs in $\mathrm{p}+\mathrm{p}(\mathrm{a})$ and in $\mathrm{Cu}+\mathrm{Cu}$ (b) collisions. The combinatorial background (mainly due to Dalitz pairs and photon conversions) derived from like-sign pairs is marked as shaded areas. Other correlated $\mathrm{e}^{+} \mathrm{e}^{-}$background is estimated to be $<10 \%$. The $\mathrm{J} / \Psi$ signal is extracted in the mass window 2.7 $<\mathrm{M}_{i n v}<3.3 \mathrm{GeV} / \mathrm{c}^{2}$. Due to the high- $\mathrm{p}_{t}$ cuts used in this study the signal-to-background ratio (S/B) is large. Figure 1(c) shows the measured $\mathrm{J} / \Psi \mathrm{p}_{t}$ spectra. It is compared to the theoretical 
calculations of NRQCD from Color Singlet (CO) and Color Octet (CS) transitions for direct J/ 's in p+p collisions (solid line) [4] and NNLO CS result (grey band) [5]. Both calculations do not include the feed-down contribution which is expected to be quite substantial. The size of the band for the CS NNLO reflects the uncertainty of scale parameters and the charm quark mass. The only calculations which seem to be close to the data points are $\mathrm{CS}+\mathrm{CO}$, but they do not include $\mathrm{J} / \Psi$ from feed down process and there seems to be very little room for an additional contribution.

It is of great importance to constrain experimentally the B-meson feed-down. STAR estimates, based on the measurements of azimuthal correlations [6] that the fraction of $\mathrm{J} / \Psi$ from B-meson decay is $13+/-5 \%$ at $\mathrm{p}_{t}>5 \mathrm{GeV} / \mathrm{c}$.
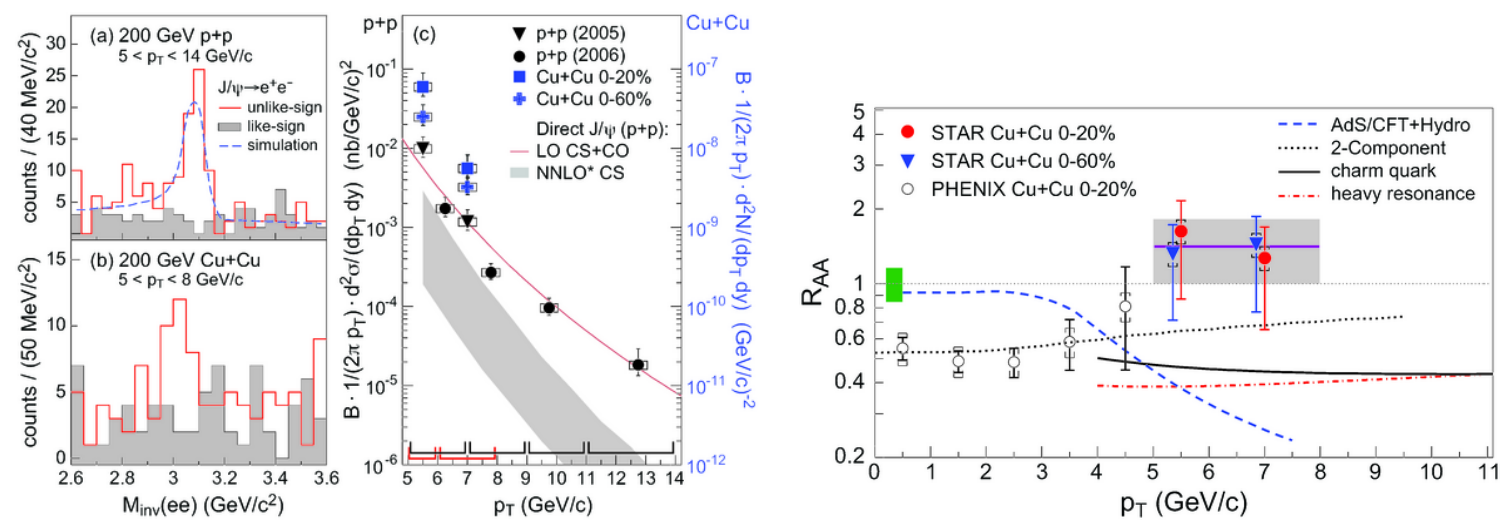

Figure 1: (left): Invariant di-electron mass distribution in (a) $\mathrm{p}+\mathrm{p}$ and (b) $\mathrm{Cu}+\mathrm{Cu}$ collisions, for opposite sign (solid) and same sign pairs (grey band) from data, and simulated $\mathrm{J} / \Psi$ peak for $\mathrm{p}+\mathrm{p}$ (dashed). $\mathrm{J} / \Psi \mathrm{p}_{t}$ distributions (c) in $\mathrm{p}+\mathrm{p}$ and $\mathrm{Cu}+\mathrm{Cu}$ at $\sqrt{S_{N N}}=200 \mathrm{GeV}$ (see text for details); (right): $\mathrm{J} / \Psi \mathrm{R}_{A A}$ as a function of $\mathrm{p}_{t}$ in in $\mathrm{Cu}+\mathrm{Cu}$ collisions. STAR data points have statistical (bars) and systematical (caps) uncertainties. The box about unity shows $\mathrm{R}_{A A}$ normalization uncertainty, which is quadratic summ of $\mathrm{p}+\mathrm{p}$ normalization and binary collision scaling uncertainties. The curves are model calculations described in the text. [3].

Figure 1(right) [3] shows the $\mathrm{J} / \Psi$ nuclear modification factor, $\mathrm{R}_{A A}$, as a function of $\mathrm{p}_{t}$ in 0 $20 \%$ in $\mathrm{Cu}+\mathrm{Cu}$ collisions from PHENIX [7] and STAR, and 0-60 \% $\mathrm{Cu}+\mathrm{Cu}$ from STAR. The data suggests that $\mathrm{R}_{A A}$ is rising towards unity for $\mathrm{p}_{t}>5 \mathrm{GeV} / \mathrm{c}$, however presence of large errors precludes the final conclusion. The average of the two STAR 0-20\% data points at high $\mathrm{p}_{t}$ is $\mathrm{R}_{A A}=1.4+/-0.4$ (stat.) +/- 0.2 (syst.). Using all STAR and PHENIX [7] points measured at high $\mathrm{p}_{t}$ lowers the value of $\mathrm{R}_{A A}$ to $1.1+/-0.3$ (stat.) +/- 0.2 (syst.). Both results are consistent with unity and differ by about two standard deviations from PHENIX measurements at lower $\mathrm{p}_{t}$. $\mathrm{J} / \Psi$ measured in $\mathrm{Cu}+\mathrm{C}$ collisions is the only hadron at top RHIC energies that does not exhibit significant suppression at high $\mathrm{p}_{t}$. It is very important to verify this finding with $\mathrm{Au}+\mathrm{A}$ (work in progress) as in those collisions a strong suppression of open charm is reported [8]. The black solid line on Figure 1(right) represents predictions for an open charm $\mathrm{R}_{A A}$ [11], and the dash-dotted line - a GLV model calculations for D-meson energy loss [12]. Both these models predict charm suppression of a factor $\sim 2$ at $\mathrm{p}_{t}>5 \mathrm{GeV} / \mathrm{c}$. This suggests that high- $\mathrm{p}_{t} \mathrm{~J} / \Psi$ production may not proceed via color-carrying channel. The dashed curve, showing an AdS/CFT-based calculation [9], also misses the data points. The two-component model (dotted line) [10], including color screening, hadronic phase dissociation, statistical recombination, formation time effects and B-meson feed- 
down, seems to describe the overall data trend well.

The $\mathrm{d}+\mathrm{Au}$ collisions are essential to understanding cold nuclear effects present in all heavy ion collisions. For the first STAR measurement, the most central $\mathrm{d}+\mathrm{Au}$ events $(0-20 \%)$ were used. Reported preliminary $\mathrm{R}_{d A u}$ is consistent with unity: $\mathrm{R}_{d A u}=1.4+/-0.6$ (stat. error only)[13]

Higher states of quarkonia family, $\Upsilon$ and its excited states, which possess significant advantages over $\mathrm{J} / \Psi$, as discussed earlier, are essential for understanding color screening in plasma [14]. The experimental data should help to constrain available QGP models [15].
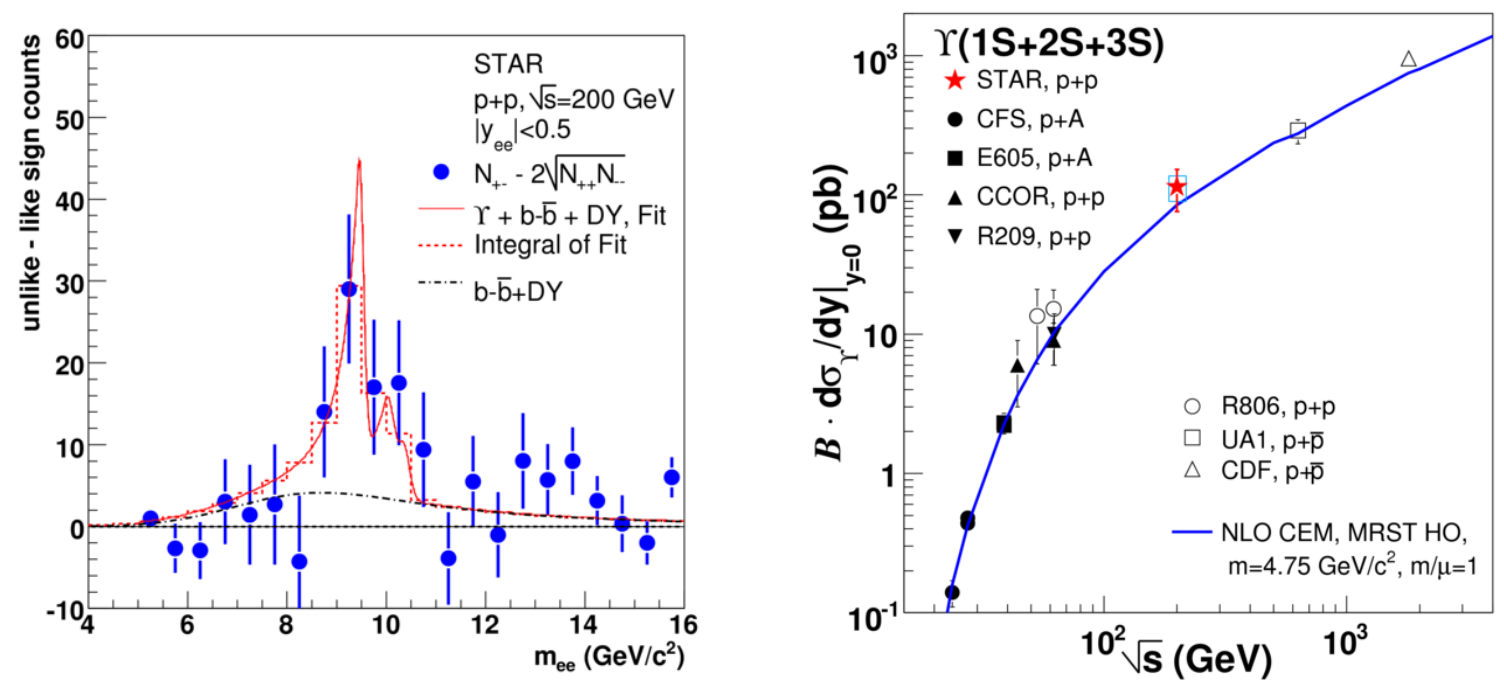

Figure 2: (left) Data used to obtain the $\Upsilon$ and continuum yields: points are the $\mathrm{e}^{+} \mathrm{e}^{-}$signal in $\mathrm{p}+\mathrm{p}$ collisions after subtraction of the like-sign combinatorial background (see text for details); (right) Evolution of the $\Upsilon(1 \mathrm{~S}+2 \mathrm{~S}+3 \mathrm{~S})$ cross section with center-of-mass energy for the world data and an NLO CEM calculation. The error bars on the STAR point are statistical and systematic. Both figures are from [17].

Besides an essential information on the deconfinement, $\Upsilon$ - family is expected to help to extract temperature of medium created in $\mathrm{A}+\mathrm{A}$ collisions. Recent results [16] indicate that almost all quarkonia states $\left(\mathrm{J} / \Psi, \Psi^{\prime}, \chi_{c}, \chi_{b}, \Upsilon(2 \mathrm{~S})\right)$ melt below $1.3 \mathrm{~T}_{c}\left(\mathrm{~T}_{c}\right.$ - critical temperature for partonhadron phase transition), and the only one to survive to higher temperature $\left(\sim 2 \mathrm{~T}_{c}\right.$ where $\mathrm{T}_{c} \sim 175$ $\mathrm{MeV})$ is the $\Upsilon(1 \mathrm{~S})$. Therefore, systematic studies of all states in $\mathrm{p}+\mathrm{p}, \mathrm{d}+\mathrm{Au}$ and $\mathrm{Au}+\mathrm{Au}$ collisions are essential.

Figure 2a shows $\mathrm{e}^{+} \mathrm{e}^{-}$signal in $\mathrm{p}+\mathrm{p}$ collisions after subtraction of like-sign combinatorial background. Error bars are statistical. Since individual states of the $\Upsilon$ family can not be separetad due to limited statistics and finite momentum resolution, this signal represents $\Upsilon(1 S+2 S+3 S)$. The fit to data (solid line) contains contributions from the $\Upsilon(1 \mathrm{~S}+2 \mathrm{~S}+3 \mathrm{~S})$ states, and contributions from Drell-Yan and b- $\bar{b}$. For details see [17]. Result obtained for cross-section [17] is consistent with the overall trend and is displayed as a function of center-of-mass energy, together with all other available data points $(p+p, p+\bar{p}$ and $p+A)$, in Figure $2 b$. The NLO CEM predictions [16] describe the data well.

Analysis of $\Upsilon$ in $\mathrm{d}+\mathrm{Au}$ collisions allowed to determine $\mathrm{R}_{d A u}^{\Upsilon}=0.98$ +/- 0.32 (stat.) +/- 0.28 (sys.), which is consistent with the binary scaling of $\Upsilon$ production. 
Preliminary results from $\Upsilon$ analysis of $\mathrm{Au}+\mathrm{Au}$ data show signal significance in the unlikesign subtracted by like-sign electron invariant mass spectrum on the level of $4 \sigma$ [18] . Improved analysis (presently in progress) will yield a measurement of $\mathrm{R}_{A A}$ with lower systematic uncertainty.

\section{Summary}

STAR has measured the $\mathrm{J} / \Psi$ and $\Upsilon(1 \mathrm{~S}+2 \mathrm{~S}+3 \mathrm{~S})$ production via the di-electron channel in $\mathrm{p}+\mathrm{p}$, $\mathrm{d}+\mathrm{Au}, \mathrm{Cu}+\mathrm{Cu}$ and $\mathrm{Au}+\mathrm{Au}$ collisons at $\sqrt{s_{N N}}=200 \mathrm{GeV}$. The $\mathrm{J} / \Psi$ nuclear modification factor $\mathrm{R}_{A A}$ in $\mathrm{Cu}+\mathrm{Cu}$ increases from low to high $\mathrm{p}_{t}$ and is consistent with no $\mathrm{J} / \Psi$ suppression for $\mathrm{p}_{t}>5$ $\mathrm{GeV} / \mathrm{c}$, in contrast to the prediction from theoretical model of quarkonium dissociation in a strongly coupled liquid using an AdS/CFT approach. The two component model with finite $\mathrm{J} / \Psi$ formation time describes the increasing trend of the $\mathrm{J} / \Psi \mathrm{R}_{A A}$.

The $\mathrm{R}_{d A u}$ of $\Upsilon(1 \mathrm{~S}+2 \mathrm{~S}+3 \mathrm{~S})$ is consistent with the number of binary scaling. Quarkonia measurements in $p+p$ and $d+A u$ will serve as a baseline for analysis in heavy ion collisions.

In the near future, with increased luminosity due to RHIC upgrades a separation of the $\Upsilon(2 S)$ and $\Upsilon(3 S)$ states should be possible.

\section{References}

[1] T.Matsui and H.Satz, Phys. Lett. B178, 4169 (1986)

[2] P.Braun-Munzinger and J.Stachel, Phys. Lett. B490, 196 (2000); L.Grandchamp and R.Rapp, Phys. Lett. B523, 60 (2001); R.L. Thews et al., Phys.Rev. C63, 054905 (2001); M.I. Gorenstein et al., Phys.Lett. B524 (2002); Yan at al., Phys.Rev.Lett. 97, 232301 (2006)

[3] B.I.Abelev et al., Phys.Rev. C80 (2009) 41902; A.Dare et al., Phys.Rev.Lett. 98, 232002 (2007); F.Abe et al., Phys.Rev.Lett. 79, 572 (1997); D.E.Acosta et al., Phys.Rev. D71, 032001 (2005)

[4] G.Nayak et al., Phys.Rev. D68, 034003 (2003)

[5] P.Artoisenet et al., Phys.Rev.Lett. 101, 152001 (2008); J.P.Lansberg - private communication

[6] W. Xie for STAR Collaboration, this proceedings

[7] A.Adare et al., Phys.Rev.Lett. 101, 122301 (2002)

[8] B.I.Abelev et al., Phys.Rev.Lett. 98 (2007) 192301; S.Adler et al., Phys.Rev.Lett. 96 (2006) 032301

[9] H.Liu et al., Phys.Rev.Lett. 98, 182301 (2007); T.Gunji, J.Phys.G: Nucl.Part.Phys. 35, 104137 (2008)

[10] X.Zhao and R.Rapp (2007), arXiv:0712.2407; Y.P.Liu et al., Phys.Lett. B678, 72 (2009)

[11] S.Wicks et al., Nucl.Phys. A784, 426 (2007)

[12] A.Adil and I.Vitev, Phys.Lett. B649, 139 (2007)

[13] C.Perkins et al., Nucl.Phys. A830 (2009) 231

[14] S.Digal et al., Phys.Rev. D64, 094015 (2001)

[15] J.F.Gunon and R.Vogt, Nucl. Phys. B 492, 301 (1997)

[16] A.Moscy and P.Petreczky, Phys.Rev. D77, 014501 (2008); A.D.Frawley et al., Phys.Rept. 462, 125 (2008)

[17] B.I.Abelev et al., arXiv:1001.2745, May 2010

[18] R.Reed for STAR Coll., Proc. of Hot Quarks 2010 Conference, June 2010, France 